\title{
Travertinos coloreados en la Cordillera Bética (SE de la Península Ibérica). Situación geológica y características petrofísicas
}

\author{
$M^{\mathrm{a}}$ Á. García-del-Cura ${ }^{(1,2)}$, D. Benavente ${ }^{(2,3)}, \mathrm{J}$. Martínez-Martínez ${ }^{(2,3)}$ and S. Ordóñez ${ }^{(2,3)}$ \\ (1) IGEO, CSIC,UCM. Facultad de Geología, c/ José Antonio Novais, 2. 28040 Madrid \\ agcura@geo.ucm.es \\ (2) Laboratorio de Petrología Aplicada. Ap 99. 03080 Alicante \\ (3) Departamento de Ciencias de la Tierra y del Medio Ambiente. Universidad de Alicante. Ap 99. 03080 Alicante. \\ (4) Instituto Geológico y Minero de España. Ríos Rosas 23, 28003 Madrid. \\ david.benavente@ua.es, javier.martinez@igme.es, salvador@ua.es
}

\begin{abstract}
RESUMEN
En la zona Bética Externa, se encuentran depósitos de travertinos coloreados de valor como rocas ornamentales cuyas características petrofísicas son poco conocidas. Están en relación con fallas activas. Las canteras de travertino se sitúan en Alhama de Almería, Albox (Almería) y Baños de Mula (Murcia).

Estos travertinos forman parte de la última etapa del relleno de las cuencas terciarias postectónicas béticas (Pleistoceno) y generalmente aparecen espacialmente asociados a episodios tobáceos.

El objetivo de este trabajo es definir las facies de estos travertinos y las características petrofísicas que presentan con vistas a su utilización como roca ornamental.

Predominan en ellos las facies bandeadas $(1-10 \mathrm{~cm})$. Su porosidad es variable $(5-15 \%)$ consistente en una microporosidad bastante homogénea, con moda $(0.005-0.05 \mu \mathrm{m})$ ligada al carbonato micrítico, y una familia de poros menos seleccionada entre $0.05-2 \mu \mathrm{m}$ asociada a cristales de mayor tamaño. La macroporosidad de tamaño y disposición variable es un rasgo característico de estos travertinos.

El coeficiente de absorción capilar es muy anisótropo y varía mucho en los diferentes travertinos estudiados. El valor mínimo encontrado en travertinos comercializados ha sido de $1.42\left(\mathrm{~g} / \mathrm{m}^{2} \mathrm{~s}^{0.5}\right)$ en dirección oblicua a la estructura en el travertino Rojo Alhama y el máximo de $10.71\left(\mathrm{~g} / \mathrm{m}^{2} \mathrm{~s}^{0.5}\right)$ en dirección paralela a la estructura en el travertino de Albox.

Su resistencia es notablemente anisótropa, encontrando valores de resistencia a compresión que pueden llegar a alcanzar $68 \mathrm{MPa}$ en dirección perpendicular al bandeado. Cortados en dicha dirección constituyen una roca ornamental de buena calidad dentro de la categoría de mármoles comerciales.
\end{abstract}

Palabras clave: Cordilleras Béticas, petrofísica, recurso minero, roca ornamental, travertino.

\section{Some colored travertines from the Betic Range (SE Iberian Peninsula): their geological setting and petrophysical properties}

\begin{abstract}
The Betic Range contains several colored travertine deposits which were formed during the last stage of the post-tectonic Tertiary-Quaternary (Pleistocene) basin refill. Associated with active faults, these travertines are quarried in Alhama de Almería, Albox (Almería) and Baños de Mula (Murcia) for subsequent use as ornamental stone.

The aim of this study was to characterize their facies and physical properties in order to establish their suitability for use as an ornamental stone.

The main facies was banded $(1-10 \mathrm{~cm})$, and the travertines presented variable porosity ranging from 515\%. Porosity is related to travertine texture: a) fairly homogeneous microporosity, with a mode between 0.005 and $0.05 \mu \mathrm{m}$, is linked to micritic carbonate; and b) a porosity mode varying widely from 0.05 to $2 \mu \mathrm{m}$ is associated with larger crystals. A characteristic feature of these travertine deposits was their macroporosity of variable size and distribution.
\end{abstract}

The coefficient of capillary absorption was highly anisotropic and presented very different magnitudes in 
García-del-Cura, M. ${ }^{a}$ Á., et al., 2017. Travertinos coloreados en la Cordillera Bética... Boletín Geológico y Minero, 128 (2): $467-483$

the different travertines studied. A minimum value of $1.42\left(\mathrm{~g} / \mathrm{m}^{2} \mathrm{~s}^{0.5}\right)$ was observed in the commercial Red Alhama travertine, in an oblique direction to the structure, while the maximum value of $10.71\left(\mathrm{~g} / \mathrm{m}^{2} \mathrm{~s}^{0.5}\right)$ was found in Albox travertine deposits, in a parallel direction to the structure. Strength was markedly anisotropic, attaining compressive strength values as high as $68 \mathrm{MPa}$ in the direction perpendicular to the bedding planes. When cut in this direction, the travertines studied yield good quality ornamental stone within the category of commercial marble.

Key words: Betic Range, mineral resource, ornamental rock, petrophysics, travertine.

\section{ABRIDGED ENGLISH VERSION}

\section{Introduction}

This paper presents a study of three varieties of colored travertine from Alhama de Almería, Albox (Almería) and Baños de Mula (Murcia), which are used as ornamental stone. The travertines from Alhama de Almería are marketed under the trade names Red, Gray Alhama and Olivillo, while those from Albox are called Yellow and/or Gold travertine. The travertine from Baños de Mula is known as Red Mula, and was used extensively in architectural heritage from the Roman times (Theater of Cartagena). Travertines are limestones with parallel layers or bedding, and four travertine facies have been defined in accordance with the features of these layers: massive facies (average layer thickness greater than $10 \mathrm{~cm}$ ), banded facies (layers between 1 and 10 $\mathrm{cm}$ ), laminated facies (commonly $0.05-1 \mathrm{~cm}$ thick) and cryptolaminated facies (millimeter layers only clearly visible under optical microscopy) (García-del-Cura et al., 2012).

\section{Objectives}

The aim of this study was to establish the suitability of these colored travertines from SE Spain for use as ornamental stone. To this end, we determined whether their petrophysical properties were suitable for use as a construction and building material. A further aim was to describe their location, facies and geological setting.

\section{Methodology}

For petrographic characterization, 30 thin sections (30 microns) of each rock type were examined using a Zeiss Assioscop transmitted light microscope. The mineralogical composition of samples was analysed by powder $\mathrm{X}$-ray diffraction on a Bruker D8 Advance diffractometer using Cu K radiation.

A detailed study of the pore structure was carried out for each rock structure and described in terms of porosity and pore size distribution. Characterization was performed using mercury intrusion porosimetry (MIP) and a helium pycnometer.

Bulk density was determined through direct measurement of dimensions, using $4 \mathrm{~cm}$ cubes. Effective porosity was measured by vacuum saturation and applying Archimedes's law, again using $4 \mathrm{~cm}$ cubes.

Real density was obtained using an AccuPyc 1330 Helium pycnometer. Water absorption by capillarity was determined using the standard method in accordance with UNE-EN-1925 (1999). Capillarity measurements were performed in different directions of samples in order to evaluate their anisotropy.

The uniaxial compressive strength test was carried out in accordance with UNE-EN 1926 (2005) using an Ibertest MEH-2000 H/FIB-50 machine. P and V ultrasonic waves were measured using a precise ultrasonic device, consisting of signal emitting-receiving equipment (Panametrics-NDT 5058PR) and an oscilloscope (TDS 3012B-Tektronix). Rock anisotropy was quantified through an ultrasonic anisotropy coefficient, Avp., which is a dimensionless coefficient defined as the ratio of the minimum to maximum P-wave velocity values measured on the three main axes of the cube-shaped samples.

More details about this methodology are given in García-del-Cura et al. (2012).

\section{Results and Discussion}

The three travertine deposits studied in this paper were formed in the geological area of the eastern Betic Ranges (Figure 1). Travertines from Alhama de Almería (Figure 2) and Albox (Figure 5) are associated with the Alpujarride Complex. Baños de Mula is located in a post-tectonic Tertiary-Quaternary basin called the ArchenaMula, which is considered a sub-basin of the Fortuna Basin (Meijninger, 2007) (Figure 7). 
The travertines (thermogene travertines, after Pentecost \&Viles, 1994) are banded limestones which are generated by $\mathrm{CO}_{2}$ originating from endogenous processes that reaches the Earth's surface via thermal sources (hot springs).

In the studied travertines, the hot springs are related to three active faults: the Albox, Alhama de Murcia (Masana et al., 2005) and Mula-Archena faults, the latter also known as the limit fault (Silva et al., 1996 and Martínez-Díaz et al., 2002).

Travertine deposits present Fe and/or Mn mineralization (García-del-Cura et al., 2007, 2008, 2009 and 2014b), which provide their typical "colored appearance".

These travertines can be considered to form a "travertine plateau" since they do not produce vertical relief; in contrast, fissure ridge travertines create a characteristic prominent topography. Plateau travertines are primarily formed through lateral progradation, whereas fissure ridge travertines grow through vertical aggradation (De Fillipis et al., 2013). Occasionally, we observed small deposits of fissure ridge travertines in Baños de Mula and Alhama de Almería.

\section{Alhama de Almería travertines}

The Alhama de Almería travertines are located in the northern zone of the Sierra de Gador Unit. The Alhama de Almería-Alicún quarries are situated in a travertine system lying over Pleistocene infill of the Almería Basin, in the Andarax River valley in the north of the Alpujarras depression or corridor (García et al., 2004)(Figure 2). Alhama travertines were formed before the Middle Pleistocene (García, 2001) and are associated with the thermal spring that historically formed the spa of Alhama (Sanchez Martos, 2001). This spring was displaced in 1522 by an earthquake associated with the fault (Silva and Rodríguez Pascua, 2014).

Alhama travertine shows "cryptolaminated facies" with a mean total porosity of $12 \%$ and a connected porosity of about $8 \%$. Mesoporosity is predominant and shows a pore size of between $100 \mu \mathrm{m}$ and $1 \mathrm{~mm}$, while fenestral macroporosity presents a pore size that ranges from $1 \mathrm{~mm}$ to more than $1 \mathrm{~cm}$.

Crystalline layers with micrite (Figure $3 \mathrm{~A}$ ) and layers with a coarse fibroradiate crystalline microfabric (Figure 3B) are present in similar proportions. "Massive facies" are also represented in Alhama travertine (a beautiful example can be seen in the travertine used in the paving at El Prat Airport Terminal, Barcelona) and are mainly composed of micrite, with a total porosity of about $8 \%$ and a connected porosity of around $6 \%$. They show a predominance of micropores ranging in size from 0.1 to $10 \mu \mathrm{m}$, corresponding mainly intercrystalline pores (Figure 9A). Their structure and physical properties are shown in Figure 4 and Table 1, respectively.

\section{Albox travertines}

The Albox travertine (Figure 5) is located in the southern part of the Sierra de Las Estancias Unit, in the Almanzora Neogene Basin, forming part of the Alpujarride Complex (Figure 1) in the Internal Zone of the Betic Range.

Here, "banded facies" predominate, with individual levels that are generally 2-3 cm thick, although they sometimes reach greater thicknesses. Millimeter laminar facies are also occasionally present (Figure 4). The massive facies presents a very marked mode of pore size, ranging from 0.1 to $1 \mu \mathrm{m}$. In laminar facies, pore size distribution is polymodal, ranging from $0.1-1 \mu \mathrm{m}$, to pores larger than $100 \mu \mathrm{m}$ (fenestral porosity) (Figure $9 \mathrm{~B}$ and $\mathrm{C}$ ). In Albox travertine, micrite shows different structures, but the pore surface is covered by fibroradiate calcite crystal, which is the most characteristic feature (Figure 6). The presence of goethite, hematite and hausmannite $\left(\mathrm{Mn}_{3} \mathrm{O}_{4}\right)$ contributes to the characteristic dark banded appearance of this stone.

\section{Red Mula Travertine}

Red Mula Travertine has historically been quarried in Baños de Mula (Murcia), mainly at the Cerro de la Almagra quarry (Figure 7).

Another important travertine outcrop in this area is located on the hill of the Fortress of Alcalá in Puebla de Mula (Murcia). Both outcrops are found in the Archena-Mula Neogene-Quaternary Basin, which is considered a sub-basin of the Fortuna Basin (Meijninger, 2007).

These travertines present predominantly "banded facies" with layer thicknesses ranging from 1 to $10 \mathrm{~cm}$, although crypto-laminations are also occasionally found (Figure 4). Iron oxyhydroxides are abundant in whole stone (Figure 8) in both primary minerals and part of the late cements.

There are several reddish-brown mineralization layers at the bottom of the Cerro de la Almagra quarry, 
García-del-Cura, M. ${ }^{a}$ Á., et al., 2017. Travertinos coloreados en la Cordillera Bética... Boletín Geológico y Minero, 128 (2): $467-483$

associated with the laminated facies. The mineralizations consist of iron and manganese oxyhydroxides, barite and celestite.

Porosity is around $13 \%$ and connected porosity is about $10 \%$. Pore distribution is polymodal, with a first pore range of $0.005-0.05 \mu \mathrm{m}$ that can be interpreted as intercrystalline between micrite crystals, a second pore mode which ranges from 0.05 to $1 \mu \mathrm{m}$ and corresponds to intercrystalline pores defined by larger crystals (mesocrystals) (Figure 8), The and a third mode greater than $2 \mu \mathrm{m}$, mainly corresponding to fenestral porosity (Figure 9D).

\section{Petrophysical Properties}

The three varieties of travertine show very similar petrophysical properties, as illustrated in Table I. In particular, Albox and Mula travertines show a marked anisotropy. Their compressive strength classifies them as moderately hard to hard rocks, according to ISRM (1981) classification. Average values of the physical properties of the rocks meet the requirements for most construction uses (López González-Mesones et al., 2001). The results obtained for ultrasonic velocities and capillary imbibition confirm the aforementioned anisotropy, which is quantified through the ultrasonic anisotropy coefficient.

Given the petrophysical properties of the studied travertines, we can conclude that they are suitable for use as a construction and building material. However, these properties show an anisotropic behavior, and thus particular attention must be paid to the direction of stone cutting and its relative placement in the building.

\section{Introducción}

Los travertinos, en la acepción más aceptada del término, son unas rocas carbonáticas generadas a partir de aguas calientes, cuyo rasgo textural fundamental es el bandeado. Este bandeado viene dado principalmente por las diferentes texturas que presenta el carbonato que lo constituye, sólo de forma ocasional se observa alguna diferencia mineralógico-geoquímica composicional. Los travertinos pueden contener los siguientes tipos de capas: capas de carbonato homogéneo de pequeño tamaño de cristal, capas micríticas; capas caracterizadas por la presencia de meso y macrocristales y capas con micrita y microesparita correspondientes a estructuras biogénicas. Estas estructuras han sido relacionadas mayoritariamente con bacterias en una superficie en contacto con agua, rica en iones carbonato, en medio continental (Gandin and Capezzuoli, 2014), mientras que a los microcristales de calcita se les suele atribuir un origen preferentemente abiótico.

Esta definición del término se ajusta al concepto de "thermogene travertines", de Pentecost and Viles (1994), como carbonatos cuyo $\mathrm{CO}_{2}$ procede de un ámbito generador endógeno que llega a la superficie a través de fuentes termales, en contraposición al concepto de "meteogene travertines" cuyo $\mathrm{CO}_{2}$ procede del suelo y que va a generar carbonatos, generalmente en relación con la fluencia de aguas relacionadas con sistemas kársticos, tanto en forma de fuentes (spring line tufas, Pedley et al., 2003) como asociados a procesos fluviales (Ordóñez et al,. 2005; Vazquez-Urbez et al., 2012; Arenas et al., 2014;
González-Martín and González-Amuchastegui, 2014, entre otros) Estos carbonatos que contienen moldes de vegetales en cantidad variable son denominados generalmente tobas o tobas carbonáticas, si bien su resistencia es menor que la de los travertinos, ocasionalmente pueden utilizarse en construcción, siendo frecuente encontrarlos en el patrimonio Arquitectónico como roca de uso estructural (sillares, sillarejos,...) (García-del-Cura et al., 2012b y 2014, González Martín et al., 2014). Es habitual que travertinos y tobas aparezcan asociados, tanto a nivel de macroescala (García-del-Cura et al., 2011, Ozkul et al., 2014) como de microescala (García-del-Cura et al., 2012 y 2012 b).

Los travertinos como material de construcción son utilizados desde la antigüedad, así tanto griegos como romanos los utilizaron como roca de uso estructural en sus templos y palacios (v.gr. Paestum en la Magna Grecia, Italia; Segesta en Sicilia; Hierapolis en Turquía y múltiples edificios de Roma, entre los que cabe destacar el Coliseo) (Pedley, 2009 y Pentecost, 2005) y también como aplacados e incluso pavimentos. Su capacidad o buena disposición para admitir enfoscados y su relativamente bajo peso y buena resistencia a compresión fueron las propiedades que jugaron mayoritariamente a su favor para su uso. Esta utilización se realizaba teniendo en cuenta sus propiedades tal como se pone de manifiesto en el estudio de Jackson et al. (2005). Estos autores encontraron valores de resistencia a compresión en travertinos utilizados como roca dimensional, o de uso estructural, en Roma de $82 \mathrm{MPa}$ (en húmedo) a $105 \mathrm{MPa}$ (en seco). Estos valores correspondían a la 
dirección perpendicular al bandeado de los travertinos. En España en el teatro romano de Cartagena fueron utilizados travertinos (Soler Huertas, 2005 y García-del-Cura et al., 2010).

Los travertinos presentan una macroporosidad muy variable que ha hecho que se hayan ensayado procesos de relleno y analizado como afectan a sus propiedades físicas. Esto puede verse en trabajos como Demirdag, 2009, que analiza diferentes procesos de relleno que denomina acabados masilla crema, resina transparente, lustrado y estucado.

Travertinos coloreados de Turquía e Irán han sido utilizados en Europa, así en Viena, en la primera mitad del siglo $X X$, encontramos referencias de Avan y Attila Red Travertine (Turquía) y Soraya Travertine (Persia). Actualmente siguen estando en el mercado con otras denominaciones como travertino Rojo Iraní. Turquía comercializa internacionalmente travertinos de diversos colores y denominaciones.

En el presente trabajo vamos a estudiar tres travertinos que han sido utilizados como roca ornamental: los procedentes de Albox y los de Alhama de Almería comercializados actualmente; y los travertinos de Mula que fueron utilizados en el Patrimonio Arqueológico y de los que se observan restos de explotación relativamente recientes.

\section{Objetivo}

El objetivo de este trabajo es contribuir al conocimiento de la potencialidad de algunos travertinos coloreados del SE español describiendo sus rasgos petrográficos principales y mostrando su ubicación geológica. Datos que pueden se utilizados como criterios de prospección de este tipo de travertinos en la región del SE de la Peninsula Ibérica.

También es un objetivo la determinación de sus propiedades petrofísicas que definen sus características y calidad como roca ornamental, lo que es una información de potencial interés para los prescriptores de la utilización de este tipo de materiales de construcción.

\section{Metodología}

Se ha realizado su estudio petrográfico con microscopia óptica de polarización, estudiando de 30- $40 \mathrm{sec}-$ ciones delgadas, de cada tipo de travertino estudiado, con un microscopio Zeiss Axioskop, así como con microscopía electrónica, utilizando un microscopio electrónico de barrido de presión variable (VPSEM)(Hittachi S3000N) para observar tanto super- ficies pulidas como superficies de fractura con sensores de electrones secundarios y de electrones retrodispersados, así como con un detector de energías dispersivas (EDS) para microanálisis (Bruker modelo XFlash 3001).

Se ha realizado una caracterización mineralógica con difracción de rayos $X$, con un difractómetro Bruker D8 Advance, con lámpara de cobre. La interpretación de los difractogramas se realizó con el programa XPowder (Martín, J.D., 2004), utilizando la base de datos ICDDPDF2.dat.

En primer lugar se caracterizó el sistema poroso de los diferentes tipos de facies con porosimetría de mercurio, y en segundo lugar, la porosidad abierta a vacío de las probetas utilizadas en los ensayos mecánicos.

El sistema poroso se caracterizó con un porosímetro de mercurio Autopore IV 9500 Micromeritics, en el rango de radios de poros de $0.002-100 \mu \mathrm{m}$. La porosidad abierta o conectada (con el método a vacío) y la densidad aparente se obtuvieron según la norma UNE EN 1936 (2007).

El transporte de agua por capilaridad se cuantifica con el coeficiente de absorción capilar, C, según la norma UNE-EN 1925 (1999), en diferentes direcciones a fin de determinar la anisotropía hídrica.

La resistencia a compresión se ha realizado según la Norma UNE-EN 1926 (2005), empleándose probetas cúbicas de $50 \mathrm{~mm}$ de lado para su determinación. Las probetas fueron secadas en estufa, a $70^{\circ} \mathrm{C}$ hasta que su masa permaneció constante, y luego enfriadas durante $24 \mathrm{~h}$ a $20^{\circ} \mathrm{C}$. El ensayo a compresión simple se realizó con una prensa lbertest MEH-2000H/FIB-50, manteniendo una velocidad de carga constante de $0.36 \mathrm{kN} / \mathrm{s}$.

Las velocidades ultrasónicas $\left(v_{p} \quad y \quad v_{s}\right)$ han sido medidas mediante el método de transmisión-recepción, según las especificaciones recomendadas por la Norma UNE-EN 14579 (2007). Se ha empleado un equipo emisor-receptor de señales (Panametrics 5058PR) conectado a un osciloscopio TDS 3012B (Tektronix). Las señales han sido transmitidas mediante dos pares de transductores polarizados (una pareja en $\mathrm{P}$ y otra en S) con frecuencia centrada en $1 \mathrm{MHz}$. Las medidas ultrasónicas se han realizado sobre las mismas probetas que posteriormente han sido rotas a compresión.

Además de la velocidad de propagación de las ondas $P$ y $S$, también se calculó el coeficiente de anisotropía ultrasónica $\left(A_{v p}\right)$. Para ello se midió la velocidad de propagación de las ondas $\mathrm{P}$ en las tres direcciones espaciales de cada probeta cúbica, y se realizó el cociente entre la velocidad mínima y la máxima registrada en cada caso, de forma que el parámetro 
está definido entre 0 y 1 , donde 1 corresponde a una roca perfectamente isótropa, y el valor disminuye conforme aumenta el grado de anisotropía

\section{Marco geológico o zona de estudio}

Los tres travertinos estudiados en este trabajo, procedentes de las canteras de Alhama y Albox, ambos de la provincia de Almería y la cantera histórica de Baños de Mula (provincia de Murcia) se han formado en el ámbito geológico de la zona de cizalla de la
Cordilleras Béticas Orientales (Silva et al. 1996), (Figura 1) dentro del Complejo Alpujárride y en relación con las fallas activas de Albox, la de Alhama de Murcia (Masana et al., 2005) y la denominada falla Límite o falla Mula-Archena (Silva et al., 1996 y Martínez-Díaz et al., 2002) que atraviesa la cuenca de Mula y tiene actividad hidrotermal asociada. Esta actividad se manifiesta en la presencia de surgencias de aguas termales de bajo grado $\left(>45^{\circ} \mathrm{C}\right)$ que han sido utilizadas históricamente, e incluso en la actualidad, como balnearios (Balneario de Alhama de Almería, Baños de Mula).

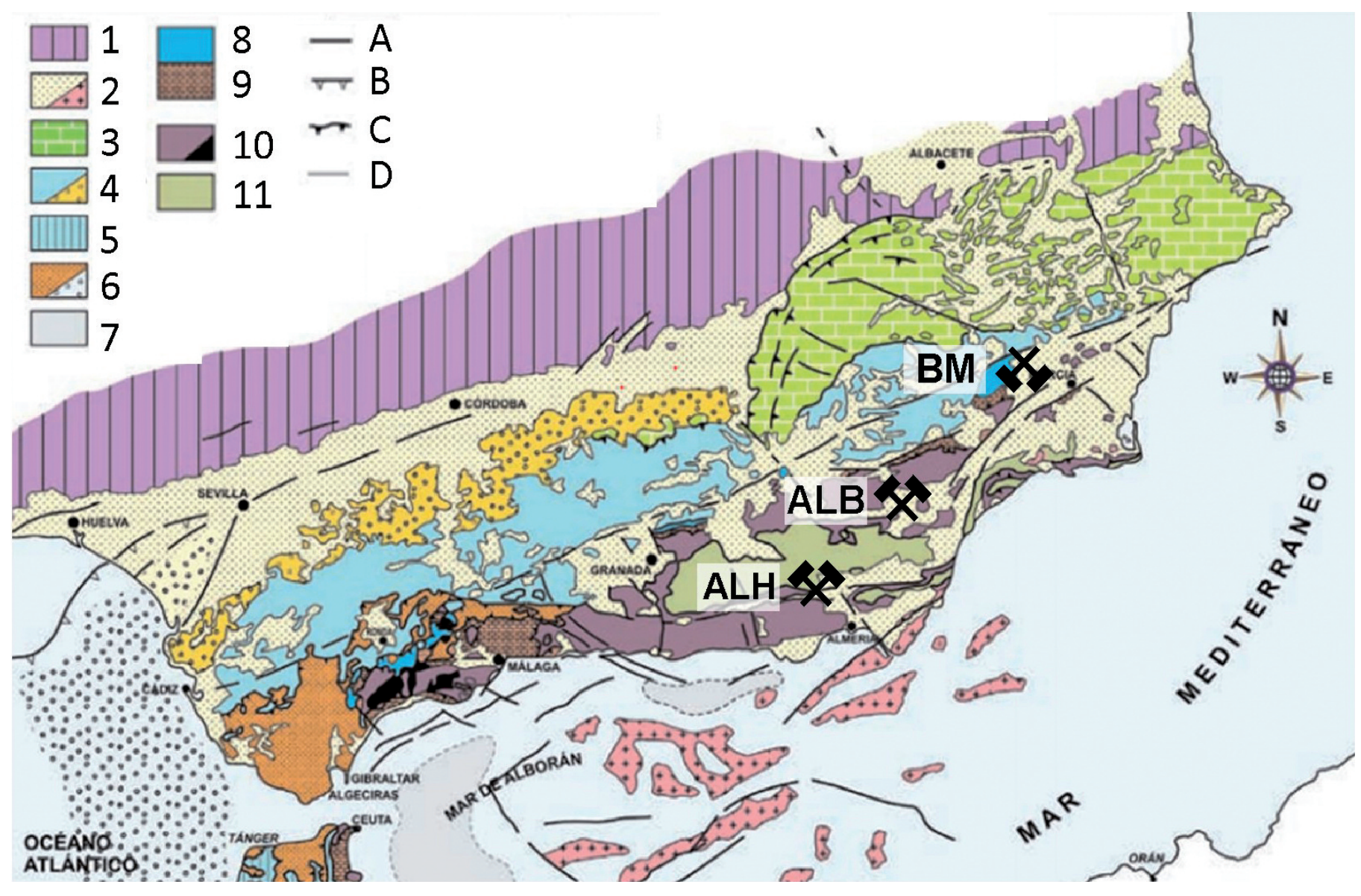

Figura 1. Mapa geológico con la situación de las zonas de canteras de travertinos estudiadas: ALH, Alhama de Almería (Almería); ALB, Albox (Almería); y BM, Baños de Mula (Murcia). Leyenda del mapa geológico: 1) Macizo lbérico; 2) Cuencas Neógenas - Cuaternarias/ Vulcanismo. Zonas Externas Béticas: 3) Prebético; 4) Subbético / Olitostromas. 5) Zona Externa del Rif. 6) Unidades de los Flysch. 7) Área de mayores espesores de sedimentos Neógenos en el Mar de Alborán. Zona Internas Béticas: 8) Complejo Dorsal; 9) Complejo Maláguide; 10) Complejo Alpujárride/Peridotitas; 11) Complejo Nevado-Filábride. Otros símbolos geológicos: A) Fallas de desgarre. B) Frente de Olitostromas. C) Cabalgamiento y fallas inversas. D Contacto discordante. Modificado de Sanz de Galdeano, C. et al., 2007.

Figure 1. Geological map showing the travertine quarries areas studied: ALH, Alhama de Almería; ALB, Albox (Almería); y BM, Baños de Mula (Murcia). Geological map captions; 1) Iberian Platform; 2) Neogene \& Quaternary Basins /Volcanism. Betic External Zone: 3) Prebetic; 4) Subbetic. 5) Rif External Zone. 6) Flysch Units. 7) Area of thicker Neogene sediments in the Alboran Sea. Betic Internal Zone: 8) Dorsal Complex. 9) Malaguide Complex; 10) Alpujarride Complex / Peridotites. 11) Nevado -Filabride Complex. Other Displayed Symbols of geological mapping: A) Strike-slip fault. B) Olitostromic front. C) Thrust or reverse fault. D) Discordant geological contact. Modified and adapted from Sanz de Galdeano el al., 2007. 
García-del-Cura, M. a Á., et al., 2017. Travertinos coloreados en la Cordillera Bética... Boletín Geológico y Minero, 128 (2): $467-483$

Este tipo de formaciones se encuentra en otros puntos en relación con la falla de Alhama de Murcia y algunas formaciones como las Carraclaca han sido utilizadas para estudiar la actividad sísmica asociada a la falla (Martínez Díaz and Hernández Enrile 2001).

En los tres travertinos objeto de este trabajo existen mineralizaciones (García-del-Cura et al., 2007, 2008,2009 y 2014b), que son las causantes del carácter "coloreado" de estos travertinos que es uno de los rasgos específicos de la mayor parte de los travertinos generados en las Cordilleras Béticas, como se manifiesta en otros travertinos como los que se encuentran en la cuenca de Tavernas (Saenz de Galdeano et al., 2008).

\section{Resultados y discusión}

El travertino de Alhama de Almería se ha comercializado como Rojo Alhama, Gris Alhama y Olivillo El travertino de Albox se comercializa básicamente como Travertino Oro y Travertino Amarillo. Ambos han sido muy utilizados, en la Península Ibérica, a finales del siglo $X X$ y principios del $X X I$, tanto en aplacados de exteriores, como en aplacados y pavimentos de interiores como es el caso del Aeropuerto del Prat donde la sala de vuelos nacionales fue pavimentada con Rojo Alhama de la cantera Travertino Antic.

El travertino de Mula no ha sido comercializado en las últimas décadas, si bien en su cantera se observan indicios de explotación correspondientes posiblemente a mediados del siglo XX. En obra romana lo encontramos en el Teatro de Cartagena existiendo otras referencias arqueológicas en zonas próximas (Soler Huertas, 2005).

Los afloramientos explotados de estos travertinos corresponden básicamente a travertinos de tipo "plateau" puesto que no tienen relieve vertical y se han formado básicamente por progradación horizontal mientras que los travertinos de tipo "fissure" crecen por progradación vertical y crean relieves con dicha dimensión predominante (De Fillipis et al. 2013). Hemos podido observar pequeños depósitos fisurales de travertinos en Baños de Mula y Alhama de Almería.

\section{Caracterizacion de los travertinos}

Vamos a tratar la situación y contesto geológico de forma individualizada y las características petrofísicas de forma conjunta lo que facilita su estudio comparativo.

\section{Travertinos de Alhama de Almería}

\section{Situación y contesto geológico}

En la zona de Alhama de Almería se pueden diferenciar varios edificios travertínicos s.l.: un edificio Norte situado en $36^{\circ} 5800,89 \mathrm{~N}, 2^{\circ} 3437,85 \mathrm{O}$, cota s.n.m. $416 \mathrm{~m} .(+169)$ y superficie de $30.000 \mathrm{~m}^{2}$ y un edificio Sur en $36^{\circ} 5747,31 \mathrm{~N}, 2^{\circ} 3435,01$ O, cota s.n.m. $476 \mathrm{~m}$. $(+229)$ y superficie de $100.000 \mathrm{~m}^{2}$. Así como un tercero al Oeste del barranco del Hormiguero situado en $36^{\circ} 5598,75 \mathrm{~N}, 2^{\circ} 3317,86$ O, cota s.n.m. 427 m. (+229 y con una superficie de $30.000 \mathrm{~m}^{2}$

Al este de Alicún se observa un lititotecto tobáceo o edificio Norte a $36^{\circ} 5811,51 \mathrm{~N}, 2^{\circ} 3528,98$ O, cota s.n.m. $456 \mathrm{~m}$ (+194, referido al Andarax), con una superficie de $100.000 \mathrm{~m}^{2}$. Y un edificio Sur a $36^{\circ} 5753,00 \mathrm{~N}, 2^{\circ} 3542,86$ O, cota s.n.m. 509 m $(+247)$ y superficie de $300.000 \mathrm{~m}^{2}$. Aquí son relativamente frecuentes los cambios laterales de facies a otros tipos de sedimentos continentales.

Las canteras de Alhama de Almería-Alicún se sitúan en un sistema travertínico que yace sobre materiales pleistocenos del relleno de la Cuenca de Almería, en la cuenca actual del Rio Andarax, en la zona norte de la denominada depresión o corredor de las Alpujarras (García et al., 2004). Los procesos tectónicos han influido tanto en la génesis de los travertinos como en su evolución posterior. Como consecuencia de este hecho encontramos grandes bloques de travertino en el seno de coluviales que han llegado a ser explotados como roca ornamental (Garcíadel-Cura et al., 2008). Es frecuente encontrar diaclasas que han experimentado procesos de reactivación en los frentes de canteras desarrollados sobre los niveles travertínicos in situ, así como estilolitos (Figura 2).

La incisión fluvial del río Andarax, que afecta a los travertinos, se inició en el Pleistoceno Medio (García, 2004) por lo que dichos travertinos se formarían con anterioridad. Sánchez Martos (2001) asoció estos travertinos con el manantial termal que constituyó históricamente los baños de Alhama y que fue afectado por un terremoto en 1522, desapareciendo de su emplazamiento (Silva \& Rodríguez Pascua, 2014). Actualmente dichas aguas son captadas a varios kilómetros y traídas al actual balneario de Alhama de Almería (Balneario de San Nicolás). Sánchez Martos (op.cit) sitúa estos travertinos sobre materiales miocenos, pliocenos y pleistocenos dispuestos sobre el Trias del complejo Alpujárride.

Los travertinos aparecen más mineralizados y por tanto más coloreados en las proximidades de la falla que condiciona la surgencia de los Baños de Alhama 

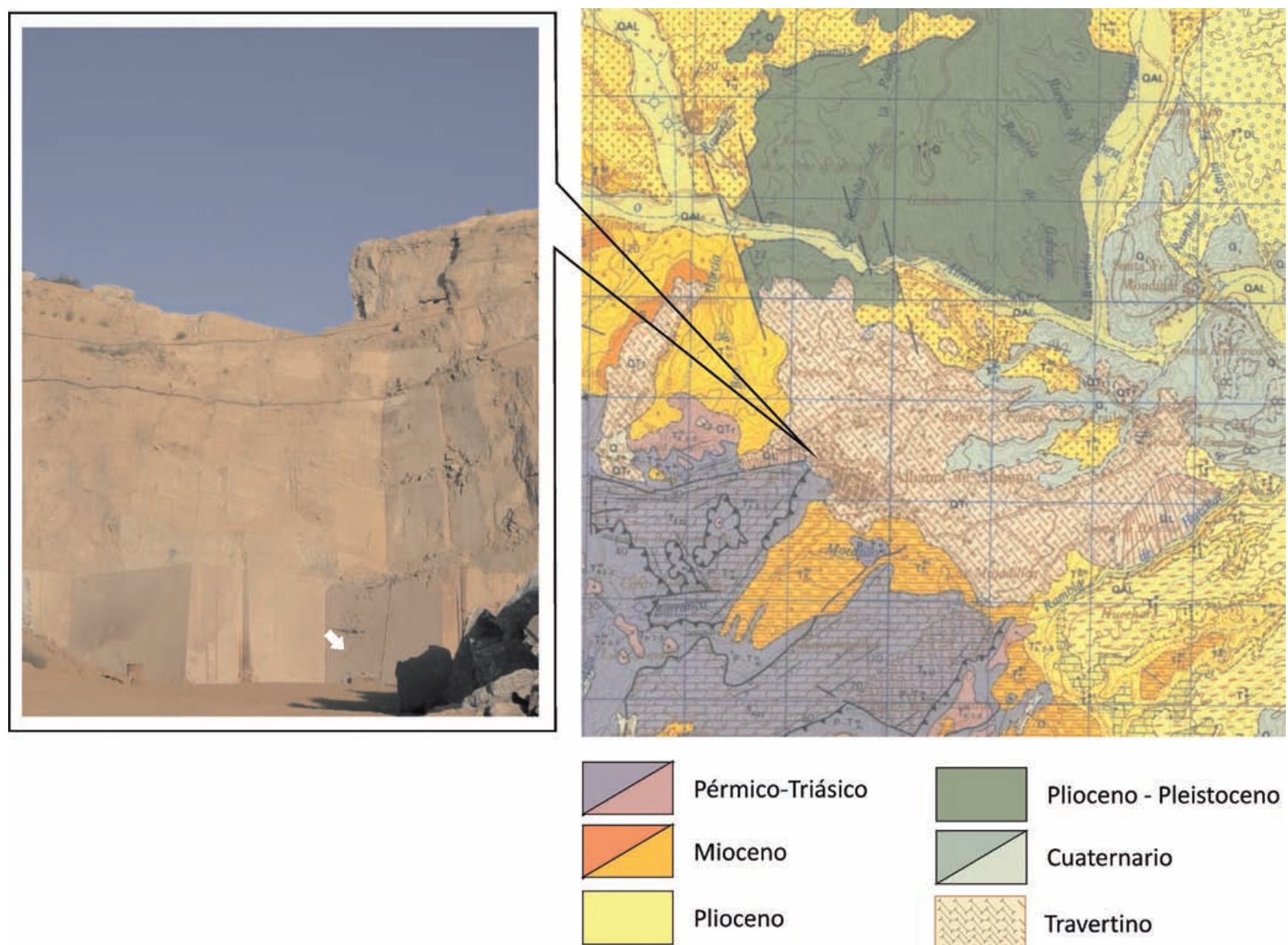

Pérmico-Triásico

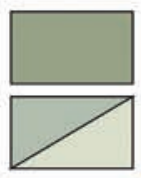

Plioceno - Pleistoceno

Mioceno

Plioceno

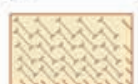

Cuaternario

Travertino

Figura 2. Detalle cantera Alhama de Almería y situación en el mapa geológico (Baena Perez and Voermans, 1977). Pueden observarse diaclasas y estilolitos (flecha).

Figure 2. Detail of Alhama de Almería quarry and location on the geological map. (Baena Perez and Voermans, 1977) Joints and stylolites (arrow) can be observed.

a la que ha afectado, desplazándola. A medida que nos alejamos de dicha falla van aumentando los moldes de plantas llegando a aparecer facies tobáceas en el seno de los travertinos explotados, que pueden alcanzar una importante proporción en la roca (García-del-Cura et al., 2012). Dichas facies tobáceas, que ocasionalmente han sido comercializadas, no han sido consideradas en el presente trabajo.

En la localidad próxima de Alicún, en las canteras existentes, vemos como hay un predominio de calizas tobáceas que en algunos puntos están atravesadas por travertinos fisurales. Los materiales tobáceos presentes en Alicún muestran abundantes moldes de tallos cuya disposición hace que sean relacionables con canales fluviales (Ordóñez and García-del-Cura, 1983 y García, 2001). Estas tobas de tallos, que presentan asociados ocasionalmente edificios estromatolíticos se encuentran en diferentes puntos, tanto en la horizontal como en la vertical, correspondiendo a sistemas tobáceos relativamente complejos parte de los cuales han sido estudiados por García, 2001 y García et al., 2003 y 2004.

\section{Caracterización petrológica - mineralógica}

Facies micríticas con texturas atribuibles a bacterias (Figura $3 \mathrm{~A}$ ) y bandas con meso y macrocristales fibrorradiados (Figura 3B) están, en general, en similar proporción en todos los puntos de estos travertinos. En los travertinos de Alhama predominan las facies bandeadas o con capas paralelas de $1 \mathrm{a} 10 \mathrm{cms}$, presentando también, con relativa frecuencia, facies masivas (capas con más de $10 \mathrm{~cm}$ de anchura), en las que pueden llegar a observarse estructuras edáficas como pedotúbulos. Son relativamente abundantes 

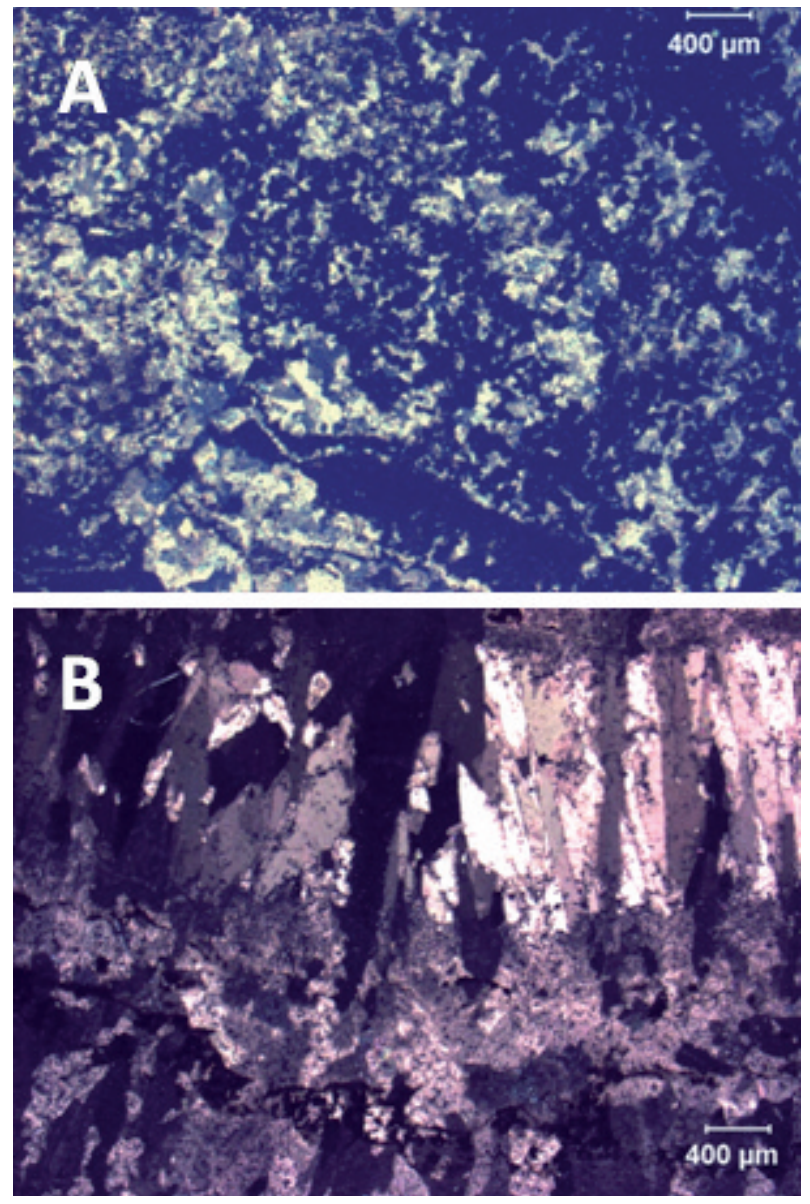

Figura 3. Fotomicrografias con nícoles cruzados de Travertinos de Alhama. A) Facies cristalina con peloides B) Láminas de cristales de calcita fibrorradiados.

Figure 3. Optical microphotographs with crossed polarized light of Alhama Travertines. A) Crystalline facies with peloids. B) Laminae of fibrorradiate calcite crystals.

las facies criptolaminadas (Figura 4). La terminología utilizada fue creada para la descripción de travertinos como rocas ornamentales (García-del-Cura et al., 2012).

El travertino está constituido básicamente por calcita de bajo contenido en magnesio. En las zonas más próximas al antiguo emplazamiento de la surgencia, donde el travertino se muestra más mineralizado, se han identificado hematites $\left(\mathrm{Fe}_{2} \mathrm{O}_{3}\right)$, goethita [$\mathrm{FeO}(\mathrm{OH})]$, cuarzo $\left(\mathrm{SiO}_{2}\right)$ y hausmanita $\left(\mathrm{Mn}^{2+} \mathrm{Mn}^{3+}{ }_{2} \mathrm{O}_{4}\right)$ y en menor cantidad pirolusita $\left(\mathrm{MnO}_{2}\right)$, baritina $\left(\mathrm{BaSO}_{4}\right)$ y celestina $\left(\mathrm{SrSO}_{4}\right)$ (García-del-Cura et al., 2008 y 2009). En las capas oscuras del bandeado encontramos acumulaciones de oxi-hidróxidos de hierro y manganeso que le dan su color característico.

\section{Características del sistema poroso}

La porosidad predominante en las facies bandeadas estudiadas por nosotros es una macroporosidad (mayor de $100 \mu \mathrm{m})$. La porosidad fenestral $(1 \mathrm{~mm}->1$ $\mathrm{cm}$ ) es paralela a la estructura predominante en la roca y contribuye a definir el bandeado. La porosidad total de estas facies es del orden del $12 \%$ y su porosidad conectada del orden de $8 \%$. En las facies masivas predomina una porosidad menor de $100 \mu \mathrm{m}(0,1-10$ $\mu \mathrm{m})$, correspondiente a poros intercristalinos, presentando una porosidad total del orden del $8 \%$ y una porosidad conectada de 6-7\% (Figura 9A). En otros trabajos realizados con estos materiales se ha corroborado que la porosidad microcristalina es la que presenta unas dimensiones más constantes, mientras que la macroporosidad puede presentar tamaños más variados (Urosevic et al. 2010 y 2011).

\section{Travertino de Albox}

\section{Situación y contesto geológico}

Las mayores masas de travertino del término de Albox se encuentran en la margen derecha de la Rambla de los Marcelinos o del Madroño una a

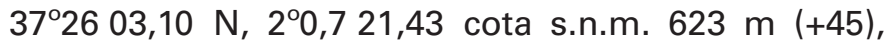
con una superficie de $45.000 \mathrm{~m}^{2} \mathrm{y}$ otra a $37^{\circ} 2557,07 \mathrm{~N}, 2^{\circ} 0734,22$ O cota s.n.m. 604 m. (+39), con una superficie de $35.000 \mathrm{~m}^{2}$. En la margen izquierda de dicha rambla encontramos dos edificios: uno a $37^{\circ} 2554,11 \mathrm{~N}, 2^{\circ} 0708,19$ O, cota s.n.m. $621 \mathrm{~m}$. $(+45)$, con superficie de $25.000 \mathrm{~m}^{2}$ y otro a $37^{\circ} 2545,99 \mathrm{~N}, 2^{\circ} 0659,53$ O, cota s.n.m. $616(+40)$ y superficie de $20.000 \mathrm{~m}^{2}$.

En la margen derecha de la Rambla del Agua Salada hay un edificio principal a $37^{\circ} 2523,36 \mathrm{~N}$, $2^{\circ} 0559,40$ O, cota s.n.m. $630 \mathrm{~m}(+50)$, con superficie de $94.000 \mathrm{~m}^{2}$ y otros dos: uno a $37^{\circ} 2529,02 \mathrm{~N}$, $2^{\circ} 0619,18$ O, cota s.n.m. $631 \mathrm{~m}(+50)$, con superficie de $15.000 \mathrm{~m}^{2} \mathrm{y}$ otro a $37^{\circ} 2519,98 \mathrm{~N}, 2^{\circ} 0620,58 \mathrm{O}$,

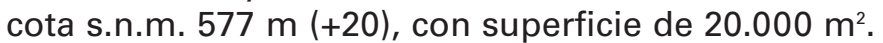

Las canteras de Albox (Figura 5) se sitúan en la Cuenca Neógena de Almanzora, limitada al Norte por la $\mathrm{S}^{\mathrm{a}}$ de las Estancias, constituida por materiales correspondientes al Complejo Alpujárride y en relación con las fallas activas de Albox (Figura 1). Los travertinos constituyen una alineación de dirección WNW-ESE bruscamente interrumpida en sus confines occidentales y orientales. Estos materiales coronan un conjunto de relieves invertidos. En ellos pueden observarse procesos de karstificacion incipientes (Figura 5). 

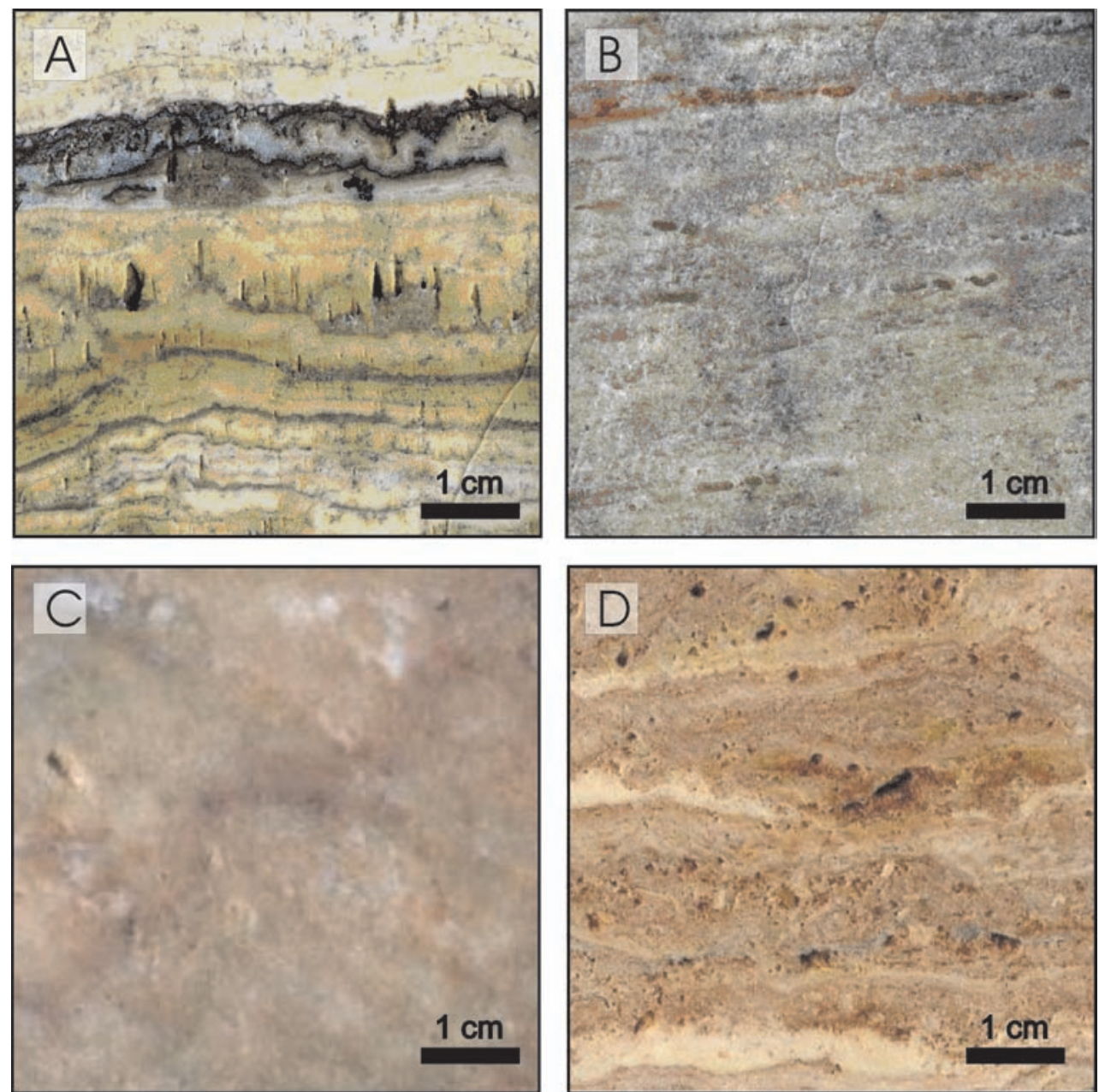

Figura 4. Ejemplos de estructuras de los travertinos: A) Laminada,Travertino de Albox, B) Criptolaminada, Travertino de Alhama, C) Masiva. Travertino de Alhama, D) Bandeada y laminada, travertino de Baños de Mula.

Figure 4. Examples of travertine structures: A Laminate structure, travertine from Albox. B Cryptolaminate structure, Travertine from Alhama. C Massive structure, travertine from Alhama, D Banded and laminate structure, travertine from Baños de Mula.

\section{Caracterizacion petrológica - mineralógica}

En estos travertinos, predominan las facies bandeadadas del orden de 2-3 cm, con algunos niveles con potencias superiores a $4 \mathrm{~cm}$ y algunas facies laminares (Figura 4). Las facies bandeadas están constituidas fundamentalmente por micrita que suele presentarse, de forma más o menos generalizada, como agregados de morfología peloidal presumiblemente de origen bacteriano (Figura 6A), siendo muy característica de los travertinos de Albox la presencia de capas y poros tapizados por mesocristales de calcita de tendencia fibrosa (Figura 6B). Las facies laminares están constituidas por alternancia de capas de micrita y de cristales fibrorradiados.

El travertino está constituido básicamente por calci- ta de bajo contenido en magnesio y algo de cuarzo (s $5 \%$ ). Las bandas mas oscuras contienen oxihidróxidos de hierro y manganeso, habiéndose detectado goethita, y en menor cantidad hausmannita, hematites y óxidos de hierro y titanio (García-del-Cura et al. 2007)

\section{Características del sistema poroso}

La porosidad en los travertinos de Albox estudiados presenta una moda muy marcada de $0,1-1 \mu \mathrm{m}$ en las facies masivas. En las facies laminares la distribución de tamaños de poros es polimodal destacando tres familias de poros: la primera próxima a $0,1 \mu \mathrm{m}$, la segunda entre 1-10 $\mu \mathrm{m}$; y la última con tamaños superiores a $100 \mu \mathrm{m}$ (Figura 9B y C). 


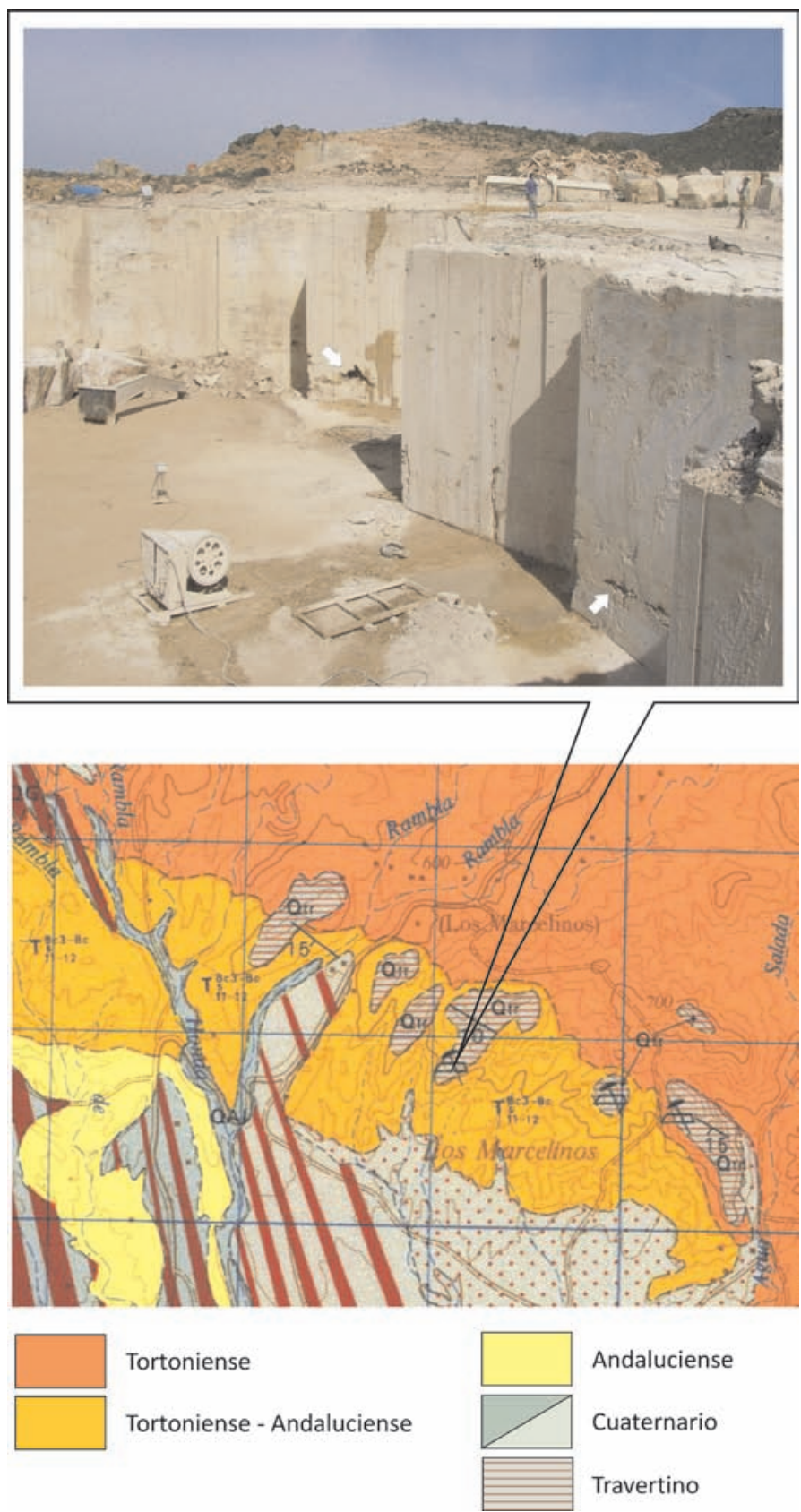

Figura 5. Cantera de Albox mostrando algunos rasgos kársticos (Flechas) y su situación en el mapa geológico (Voermans et al., 1972). Foto de J.A. González Martín.

Figure 5. Albox quarry showing some karstic features (arrows) and location on the geological map (Voermans et al., 1972). Photograph by J.A. González-Martín.

Valores similares de porosidad en travertinos de Albox encontraron Molina et al. (2011) definiendo varias familias de poros en los siguientes rangos de tamaños: $1-10 \mu \mathrm{m}, 20-50 \mu \mathrm{m}$ y > $100 \mu \mathrm{m}$.

\section{Travertino de Mula}

\section{Situación y contesto geológico}

Los travertinos explotados históricamente proceden básicamente de la cantera del Cerro de la Almagra en el término de Baños de Mula (provincia de Murcia) (Figura 7). Se encuentran en la margen izquierda del río Mula en una cota $256-257$ s.n.m. $(+50 \mathrm{~m})$, con coordenadas $38^{\circ} 0214,59 \mathrm{~N}, 1^{\circ} 2558,57 \mathrm{O}, \mathrm{y}$ una superficie que supera los $30.000 \mathrm{~m}^{2}$

Existen más afloramientos de travertinos en la zona, siendo especialmente dignos de mención por su visibilidad los que coronan el cerro en el que se encuentra la Fortaleza de Alcalá, en el término de Puebla de Mula, cota 364 m s.n.m. (+100 m), con coordenadas $38^{\circ} 0152,04 \mathrm{~N}, 1^{\circ} 2617,10 \mathrm{O}$ y una extensión aproximada de $600 \mathrm{~m}^{2}$.

Ambos afloramientos son Bien de Interés Cultural (BIC) de la Región de Murcia por razones arqueológicas e históricas.

Estos afloramientos se encuentran en la denominada cuenca Neógeno-Cuaternaria de Archena - Mula (Loiseau et al., 1990), considerada una subcuenca de la cuenca de Fortuna (Meijninger, 2007). Se trata de una cuenca postectónica ubicada en una zona con una neotectónica activa (Martínez-Díaz et al., 2002), una de cuyas manifestaciones actuales serían las fuentes termales existentes en la zona (García-delCura et al., 2014).

\section{Caracterización petrológica - mineralógica}

En estos travertinos predominan las facies bandeadas, con grosores de $1-10 \mathrm{~cm}$, mostrando en algunos puntos facies criptolaminadas (Figura 4). En la parte superior del frente de cantera del Cerro de la Almagra hemos observado facies laminadas asociadas a mineralizaciones que hemos determinado como de oxihidróxidos de hierro y manganeso, con presencia de sulfatos de bario y estroncio (barita y celestina) e indicios de arsénico.

Las bandas presentan predominantemente texturas meso y macrocristalinas con cristales isométricos, fibrosos y fibrosorradiados (Figura 8) las bandas más finas son capas micríticas con texturas que pueden ser relacionables con bacterias, como morfologías peloidales (Figura 8A) (Chafetz and Folk, 1984 y Chafetz and Guidry, 1999). Es frecuente observar varias etapas de relleno de poros (Figura 8b). En algunos puntos llegan a aparecer estructuras equiparables a estromatolitos. 

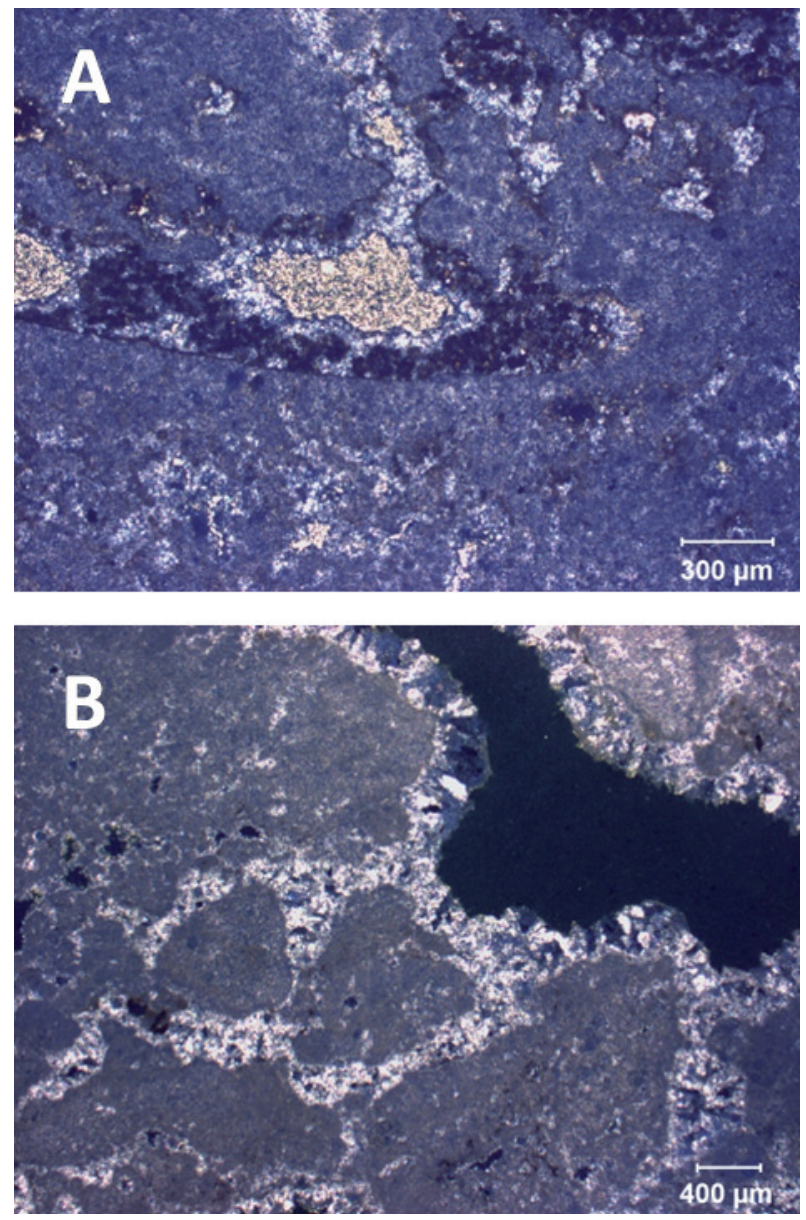

Figura 6. Fotomicrografías de travertino de Albox mostrando poros tapizados por cristales de calcita A) Con nícoles paralelos, micrita, peloides, anteriores al relleno y mesocristales. B) Tapiz de cristales de calcita fiborradiados en poro. Nícoles cruzados.

Figure 6. Optical microphotographs (MOP) of travertine from Albox, showing pores covering by calcite crystals A) Parallel polarized light. Micrite, peloids and mesocrystals. B) Crossed polarized light. Pore skin of fibrorradiate calcite crystals.

\section{Características del sistema poroso}

Su porosidad es del orden de $10 \%$. Su sistema poroso muestra una distribución polimodal de tamaño de poro (Figura 9D), donde existe una moda relacionada con los poros intercristalinos que presenta la micrita con poros del orden de $0,01 \mu \mathrm{m}(0,005-0,05 \mu \mathrm{m})$ otra asociada a los mesocristales de calcita $(0,05-2 \mu \mathrm{m})$ y una última, la de mayor tamaño $(>2 \mu \mathrm{m})$ correspondiente a porosidad fenestral mas o menos rellena por algunas fases cementantes (oxihidróxidos de hierro y/o calcita)
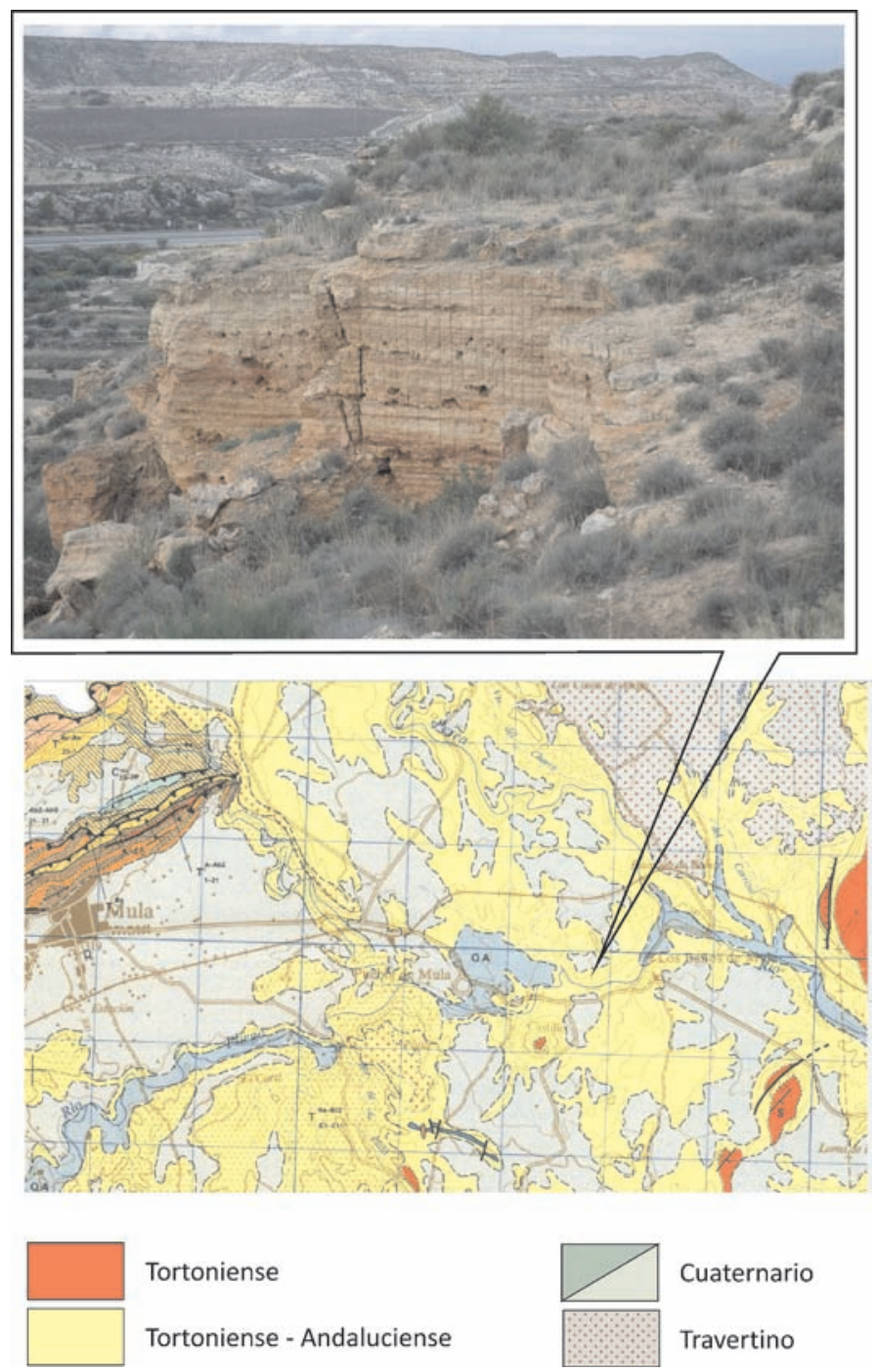

Figura 7. Frente de Cantera del Cerro de La Almagra (Baños de Mula) mostrando huellas de explotación y situación en el mapa geológico (Jerez Mir et al., 1974).

Figure 7. Quarry face of the Cerro de la Almagra (Baños de Mula) showing traces of exploitation and location on the geological map (Jerez Mir et al. 1974).

\section{Propiedades petrofisicas}

Las propiedadaes petrofísicas de los travertinos estudiados muestran unos rasgos generales y otros específicos de cada uno de los travertinos (Tabla 1). Algo similar a lo que ocurría con la porosidad, en la que el rasgo característico era el carácter bimodal o polimodal de los tamaños de los poros, variando sustancialmente el volumen de los poros de menos tamaño (Figura 9).

El coeficiente de absorción capilar es muy anisótropo y presenta magnitudes muy distintas en los 

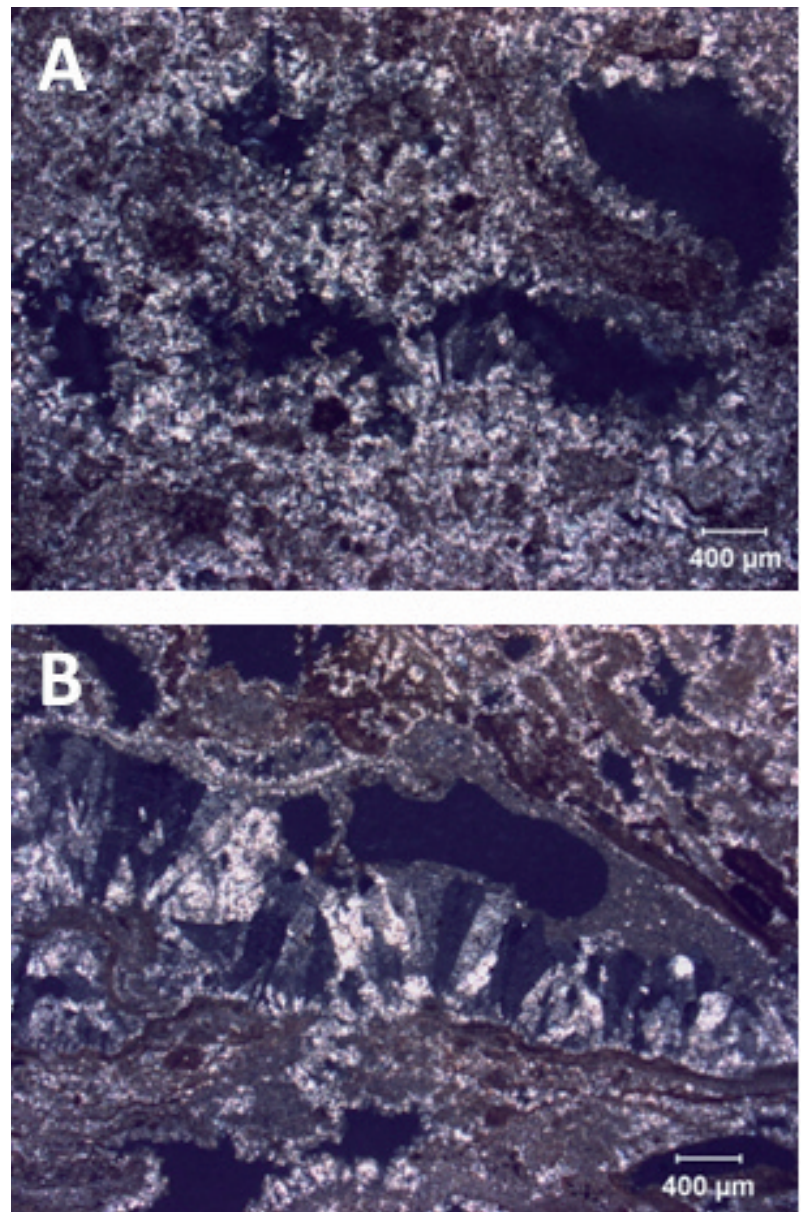

Figura 8. Fotomicrografias con nícoles cruzados del travertino de Baños de Mula mostrando calcita microcristalina y oxihidróxidos de hierro. A) Microcristales y peloides B) Láminas micríticas y microcristalinas y poro fenestral parcialmente relleno de calcita fibrorradiada.

Figure 8. Optical microphotographs (MOP) with crossed polarized light of Baños de Mula travertine showing microcrystalline calcite and Fe-oxihydroxides. A) Microcrystals and peloids. B) Micrite and microcrystals laminae and a fenestral pore partially filled by fibrorradiate calcite crystals.

diferentes travertinos estudiados, en función del tamaño de poro y su conectividad, el valor mínimo encontrado en travertinos comercializados ha sido de $1.42 \mathrm{~g} / \mathrm{m}^{2} \mathrm{~s}^{0.5}$ en dirección oblicua a la estructura en el travertino Rojo Alhama y el máximo de $10.71 \mathrm{~g} / \mathrm{m}^{2} \mathrm{~s}^{0.5}$ en dirección paralela a la estructura en el travertino de Albox.

En la dirección paralela, las facies bandeas muestran un transporte diferencial en función de la distribución de tamaño de poros que presenten, su porosidad y conectividad entre los poros. Así, cuando los poros tienen un tamaño entre 1-100 micras presentan valores de coeficiente de absorción capilar mayores que si presentan un tamaño menor.
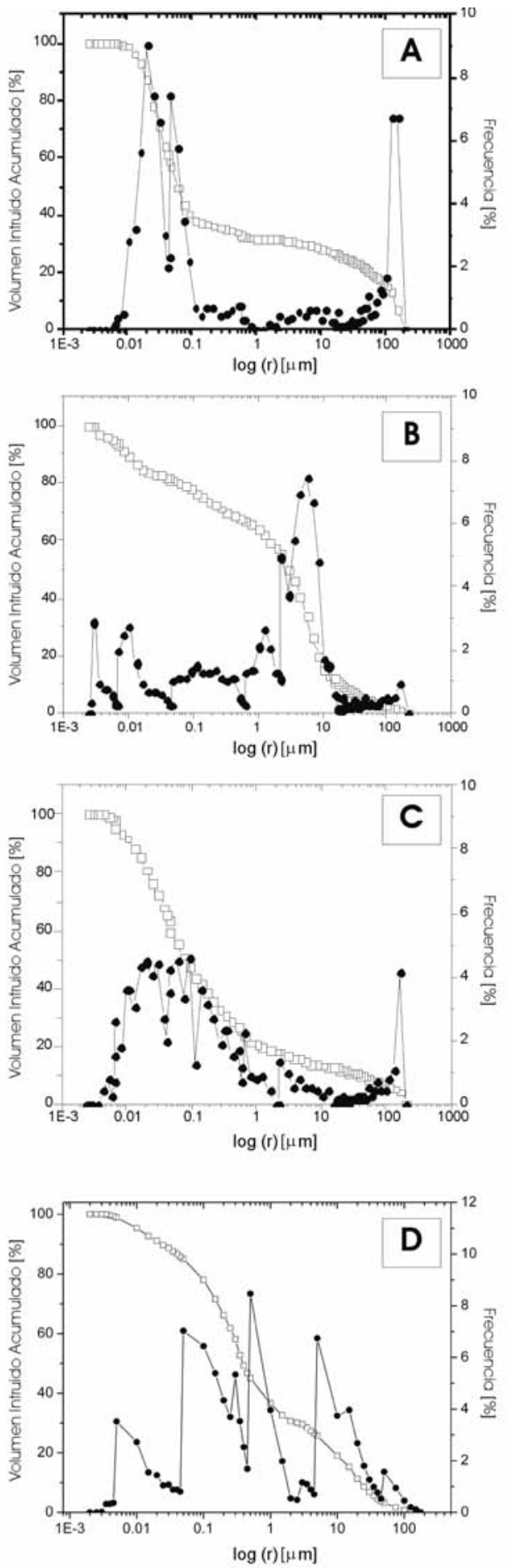

Figura 9. Ejemplo de distribución de tamaño de poros de travertinos coloreados del SE de la Peninsula Ibérica: A) Alhama de Almeria; B) Albox, cantera Los Marcelinos; C) Albox, cantera Eurostone; D) Cantera de La Almagra (Baños de Mula); es patente el caracer polimodal de todas las porosimetrias.

Figure 9. Examples of pore size distribution of colored travertine from SE of Iberian Peninsula; A) Alhama de Almeria; B) Albox, Los Marcelinos quarry; C) Albox, Eurostone quarry; D) La Almagra quarry (Baños de Mula). The polimodal character of all pore size distribution is clear. 
García-del-Cura, M. ${ }^{a}$ Á., et al., 2017. Travertinos coloreados en la Cordillera Bética... Boletín Geológico y Minero, 128 (2): $467-483$

\begin{tabular}{|c|c|c|c|c|c|}
\hline \multirow{2}{*}{ PROPIEDADES } & \multirow{2}{*}{$\begin{array}{l}\text { Travertino de Alhama } \\
\text { (Facies criptolaminada) } \\
\text { Oblicuo a la estructura }\end{array}$} & \multicolumn{2}{|c|}{$\begin{array}{c}\text { Travertino de Albox } \\
\text { (facies masivas-bandeadas porosas) }\end{array}$} & \multicolumn{2}{|c|}{$\begin{array}{l}\text { Travertino de Mula } \\
\text { Facies laminadas }\end{array}$} \\
\hline & & $\begin{array}{c}\text { Paralelo } \\
\text { a la estructura }\end{array}$ & $\begin{array}{l}\text { Perpendicular } \\
\text { a la estructura }\end{array}$ & $\begin{array}{c}\text { Paralelo } \\
\text { a la estructura }\end{array}$ & $\begin{array}{l}\text { Perpendicular } \\
\text { a la estructura }\end{array}$ \\
\hline Densidad $\left(\mathrm{g} / \mathrm{cm}^{3}\right)$ & $2.51 \pm 0.01$ & \multicolumn{2}{|c|}{$2.34 \pm 0.06$} & \multicolumn{2}{|c|}{$2.33 \pm 0.05$} \\
\hline Porosidad (\%) & $5.48 \pm 0.43$ & \multicolumn{2}{|c|}{$11.82 \pm 4.05$} & \multicolumn{2}{|c|}{$10.01 \pm 1.40$} \\
\hline $\begin{array}{l}\text { Resistencia a compresión } \\
\text { (MPa). }\end{array}$ & $49.48 \pm 10.36$ & $43.18 \pm 14.07$ & $36.82 \pm 3.70$ & $57.24 \pm 10.51$ & $44.64 \pm 6.09$ \\
\hline $\mathrm{Vp}(\mathrm{m} / \mathrm{s})$ & $4073.53 \pm 112.30$ & $5629.26 \pm 154.02$ & $5286.92 \pm 112.46$ & $5526.01 \pm 156.82$ & $5312.54 \pm 152.75$ \\
\hline Vs $(\mathrm{m} / \mathrm{s})$ & $2234.94 \pm 17.41$ & $3065.15 \pm 73.87$ & $2934.32 \pm 56.32$ & $2992.33 \pm 76.87$ & $29173.57 \pm 84.61$ \\
\hline $\begin{array}{l}\text { Coeficiente de anisotropía } \\
\text { ultrasónica (adimensional) }\end{array}$ & $0.85 \pm 0.08$ & \multicolumn{2}{|c|}{$0.92 \pm 0.03$} & \multicolumn{2}{|c|}{$0.96 \pm 0.03$} \\
\hline
\end{tabular}

Tabla I. Propiedades petrofísicas de travertinos coloreados del SE de la Península Ibérica.

Table I. Petrophysical properties of colored travertines from SE of Iberian Peninsula.

Respecto a las propiedades mecánicas, las tres variedades de travertino muestran un comportamiento bastante similar. El valor medio de resistencia a compresión es equiparable en los tres casos, siendo ligeramente superior en el Travertino de Mula (facies laminadas), y presentando valores menores en el Travertino de Albox (facies masivas y bandeadas porosas), clasificándose en los tres casos como rocas moderadamente duras a duras según la clasificación ISRM (1981). Aunque los valores de resistencia a compresión de los travertinos estudiados están lejos de las características mecánicas de una roca ornamental sedimentaria de gran calidad como el Crema Marfil $\left(\sigma_{c-\text { saturada }}=120 \mathrm{MPa}\right.$, según Ordóñez et al. 1996; $\sigma_{\mathrm{c} \text {-seco }}=$ $135 \mathrm{MPa}$, según López Buendía et al., 2013), cumplen sobradamente los requerimiento para la mayor parte de los usos constructivos (López González-Mesones et al., 2001).

Las probetas de los travertinos de Albox y de Mula han sido ensayadas en dirección paralela y perpendicular a la estructura. En ambos casos se ve como los valores de la resistencia a compresión son más altos si los esfuerzos se aplican en dirección paralela al bandeado/laminación de la roca, llegando a encontrar valores de resistencia a compresión que pueden Ilegar a alcanzar $68 \mathrm{MPa}$ en dicha dirección. Ésto se asocia directamente a la distribución heterogénea del sistema poroso. En las facies laminadas/bandeadas, los poros se distribuyen en bandas paralelas de forma que la estructura resulta dividida en tramos con una concentración de poros variable. Cuando el esfuerzo se transmite por la roca paralelamente a estas bandas, la sección efectiva de la probeta a diferentes alturas siempre contiene un porcentaje semejante de tramos porosos y tramos poco porosos. Estos últimos ofrecen mayor resistencia mecánica que los primeros por lo que actúan como "soportes estructurales" de la probeta soportando y trasmitiendo las cargas a través de ella (García-del-Cura et al., 2012). En cambio, cuando el esfuerzo se trasmite perpendicularmente a la estructura, la sección efectiva de la probeta está formada en su totalidad por tramos porosos o tramos poco porosos, según la altura de la probeta que consideremos. Los tramos porosos resultan mucho menos resistentes por lo que colapsan mecánicamente mucho antes que los tramos poco porosos, generando una inestabilidad que acaba provocando la rotura general de la probeta.

La porosidad es considerada el principal factor petrográfico condicionante de la resistencia mecánica de una roca. Sin embargo, las tres variedades de travertino estudiadas presentan una resistencia a compresión similar, aunque la porosidad delTravertino de Alhama estudiado es sensiblemente menor que las del Travertino de Albox y de Mula. Ésto es debido a que el tamaño de cristal es otro parámetro influyente en el comportamiento mecánico (Martínez-Martínez et al., 2011). El travertino de Alhama posee, de media, un tamaño de cristal sensiblemente mayor (entre 50 y $200 \mu \mathrm{m})$ a los otros dos $(<20 \mu \mathrm{m})$. Esto justifica el hecho de que finalmente muestre una resistencia menor a la que cabría esperar si sólo se tuviese en cuenta la porosidad de las muestras.

Respecto a la resistencia a flexión los travertinos coloreados muestran valores del orden de $11.5 \mathrm{MPa}$ para el Travertino Amarillo, según catálogo y para el Travertino Rojo, en el Anuario de la Piedra Natural de 2012, se dan valores de resistencia a flexión de 10.1 $\mathrm{MPa}$, habiendo determinado nosotros anteriormente (García-del-Cura et al., 2008) 7.8 $\pm 1.3 \mathrm{MPa}$. Estos valo- 
res son del orden de los que presentan otras rocas ornamentales comerciales, como el Crema Marfil antes citado (Ordóñez et al., 1996).

La velocidad de propagación de las ondas ultrasónicas registrada en estas rocas es similar a las velocidades medidas por otros autores en rocas similares (Török, 2008; Török and Vásárhelyi, 2010; Demirdag, 2009). Comparando las velocidades medidas en las tres variedades de travertino estudiados en este trabajo, vemos que el de Mula y el de Albox ofrecen valores de $v_{p} y v_{s}$ superiores al de Alhama. El mayor tamaño de cristal de esta variedad justifica, de nuevo, la menor velocidad de propagación de las ondas, y provoca que a pesar de su baja porosidad, el travertino de Alhama sea la roca que peores características ultrasónicas muestre. La importancia del tamaño de cristal en la propagación de las ondas también fue destacada por Demirdag (2009).

Las estructuras bandeadas y/o laminadas de los travertinos tienen una baja influencia en la propagación de las ondas ultrasónicas. Tanto el travertino de Mula como el de Albox registran valores mínimos en $v_{p}$ y $v_{s}$ cuando son medidos perpendicularmente a la estructura. Esto es debido al efecto pantalla que provoca la distribución de la porosidad en bandas paralelas (Martínez-Martínez et al., 2011). Sin embargo, la disminución en la velocidad generada por la distribución de la porosidad en bandas no es especialmente significativa, ya que al calcular el coeficiente de anisotropía ultrasónica (Tabla I) vemos que los valores son muy próximos a 1 (valor asociado a una roca perfectamente isótropa).

\section{Conclusiones}

El contenido en oxihidróxidos de hierro y/o manganeso, origen de la coloración de los travertinos estudiados, experimenta variaciones, disminuyendo a medida que aparecen facies tobáceas que han podido desarrollarse al enfriarse las aguas, ya que siempre se sitúan en lugares mas alejados del presumible punto de surgencia de las aguas.

Los edificios travertínicos estudiados, en especial los de tipo "Manto" constituyen la última etapa (Pleistoceno) del relleno de las cuencas terciarias postectónicas, de la zona Externa de la Cordillera Bética, en el dominio Alpujárride, apareciendo en ocasiones asociados a depósitos tobáceos. Estos edificios travertínicos están en relación con procesos hidrotermales asociados a fallas activas.

Las tres variedades locales de travertino estudiadas muestran un comportamiento mecánico relativamente similar que incluye una notable anisotropía. En general cumplen los requerimientos para su utilización en obra, en los diferentes elementos constructivos, siempre que se tenga en cuenta la anisotropía de sus propiedades a la hora de su colocación, y ésta se haga utilizando la dirección más favorable, es decir más resistente frente a los esfuerzos.

La relación existente entre la porosidad y la resistencia a compresión de los travertinos estudiados pone de manifiesto que la porosidad no es el único factor que influye en la resistencia de la roca y su consiguiente anisotropía. La estructura y la textura de la roca, la cual incluye la morfología y tamaño de los poros así como el tamaño de cristal, sus dimensiones y distribución son factores que también contribuyen a determinar la anisotropía de las propiedades petrofísicas de estos travertinos.

\section{Agradecimientos}

A Nora Cueto por su colaboración en el estudio del comportamiento hídrico de algunos materiales. Este trabajo ha contado con la ayuda del MEC: Proyecto CGL2006-05027y de la Comunidad de Madrid: Proyectos Geomateriales (S2009/Mat-1629 y S2023/MIT 2914).

\section{Rererencias}

Arenas, C., Vázquez-Urbez, M., Pardo, G. and Sancho, C. 2014 Sedimentology and depositional architecture of tufas deposited in stepped fluvial systems of changing slope: Lessons from the Quaternary Añamaza valley (Iberian Range, Spain) Sedimentology, 61, 133-171.

Baena Perez, J. and Voermans, F. 1977. Mapa Geológico de España 1:50.000, hoja $n^{\circ} 1044$ Alhama de Almería. IGME. Madrid.

De Filippis, L., Faccenna, C., Billi, A., Anzalone, E., Brilli, M., Soligo, M. and Tuccimei, P. 2013. Plateau versus fissure ridge travertines from Quaternary geothermal springs of Italy and Turkey: Interactions and feedbacks between fluid discharge, paleoclimate, and tectonics. EarthScience Reviews, 123, 35-52.

Demirdag, S. 2009. The effect of using different polymer and cement based materials in pore filling applications on technical parameters of travertine stone. Construction and Building Materials, 23, 522-530.

Gandin, A. and Capezzuoli, E. 2014. Travertine: Distinctive depositional fabrics of carbonates from thermal spring systems. Sedimentology, 61, 264-290.

García, A.F., 2001. Quaternary stream incision and topographic development in the eastern Alpujarran Corridor, Betic Cordillera, southern Spain (Almería). PhD thesis, The University of California, Santa Barbara. 214 pp.

García, A. F., Zhuc, Z., Ku, T.L., Sanz de Galdeano, C., Chadwick, O.A. and Chacón Montero. J. 2003. 
García-del-Cura, M. ${ }^{a}$ Á., et al., 2017. Travertinos coloreados en la Cordillera Bética... Boletín Geológico y Minero, 128 (2): $467-483$

Tectonically driven landscape development within the eastern Alpujarran Corridor, Betic Cordillera, SE Spain (Almería). Geomorphology, 50, 83- 110.

García, A. F., Zhuc, Z., Ku, T.L., Chadwick, O.A. and Chacón Montero. J. 2004. An incision wave in the geologic record, Alpujarran Corridor, southern Spain (Almería) Geomorphology, 60, 37 - 72.

García-del-Cura, M.A., La Iglesia, A., Benavente, D., Bernabeu, A. and González Martín, J. A. 2007. Mineralogía de los travertinos pleistocenos de Albox (Almería), importante recurso de materia prima de rocas ornamentales. Macla, 7, 89.

García-del-Cura, M.A., La Iglesia, A., Ordóñez, S., SanzMontero, M. E. and Benavente, D. 2008. Óxidos de hierro y manganeso en travertinos de Alhama de Almería. Macla, 9, 107-108.

García-del-Cura, M.A., Sanz Montero, M.E., Benavente D., Martínez-Martínez J., Bernabéu A., Cueto, N. 2008. Sistemas travertínicos de Alhama de Almería: características petrográficas y petrofísicas. Geo-Temas 10: 15551558.

García-del-Cura, M.A., Sanz-Montero, M.E., La Iglesia, A. and Ordóñez, S. 2009 Carbonate facies and sedimentological model of travertine-tufa quaternary deposits in the Betic Cordillera (SE Spain). $27^{\text {th }}$ IAS Meeting of Sedimentology Alghero, Italy. 20th -23rd September 2009. p. 518.

García-del-Cura, M.A, Benavente, D., Martínez Martínez, J. and Rodríguez García, M.A. 2010. Travertinos: del Imperio Romano al siglo XXI. Del Teatro Romano de Carthago Nova al aeropuerto de El Prat. Roc-Maquina. Mayo-Junio 2010, 124, 46-49.

García-del-Cura, M.A., Benavente, D., Martínez-Martínez, J. and Cueto, N. 2011. Sedimentological relationship between carbonate tufa and travertine building stones. In: Abstracts, 28 th IAS Meeting of Sedimentology, 5- 8 Julio Zaragoza, Spain (Eds. B. Bádenas, M. Aurell and A.M. Alonso-Zarza), p. 115.

García-del-Cura, M.A., Benavente, D., Martínez-Martínez, J. and Cueto, N. 2012. Sedimentary structures and physical properties in travertine and carbonate tufa building stone. Construction \& Building Materials, 28, 456-467.

García-del-Cura, M.A., Benavente, D. and MartínezMartínez, J. 2012b. Tobas y travertinos en el patrimonio arquitectónico de Valencia: propiedades físicas y durabilidad. VIII Congreso Geológico de España, Oviedo, Julio 2012. Publicado en Geotemas 13: 1044-1047.

García-del-Cura, M.A., Benavente, D. and MartínezMartínez, J. 2014. Las tobas: un recurso pétreo. En González-Martín J.A. and González Amuchastegui, M.J. Las acumulaciones tobáceas en España. Sociedad Española de Geomorfología. Badajoz. Cap 25: 349-355.

García-del-Cura, M.A., Benavente, D., Martínez-Martínez, J. and Ordóñez, S. 2014b. Los travertinos de Baños de Mula (Murcia). Una roca de interés sedimentológico y arqueológico. Geogaceta 56: 75-78.

González, J.A., Fidalgo, C., Arteaga, c., González, M.J. and Rubio, V. 2014. La degradación antrópica de los paisajes tobáceos. En González-Martín J.A. and González
Amuchastegui, M.J. Eds Las acumulaciones tobáceas en España. Sociedad Española de Geomorfología. Badajoz Cap 23; 317-338.

González-Martín J.A. and González Amuchastegui, M.J. 2014 Las acumulaciones tobáceas en España. Sociedad Española de Geomorfología. Badajoz, 418 pp.

ISMR (1981). Rock characterization. Testing and monitoring. ISRM suggested methods. Brown, E.T. Ed. Commision on testing and monitoring. International Society for Rock Mechanics, Pergamon Press, 211 pp.

Jackson, M.D, Marra, F., Hay R.L., Cawood, C., and Winkler, E.M. 2005. The judicious selection and preservation of tuff and travertine building stone in ancient Rome. Archaeometry, 47, 485-510.

Jerez Mir, L., Jerez Mir, F. y García-Monzón, G. (1974). Mapa Geológico de España 1:50.000, hoja no 912 (Mula). IGME, Madrid.

López Buendía, A., Guillém, C., Cuevas, J.M., Mateos, F. and Montoto, M. 2013. Natural stone reinforcement of discontinuities with resin for industrial processing. Engineering Geology, 166, 39-51.

López González-Mesones, F., Escribano Villan, J. and Nieves Aguirre, G. 2001. Manual para el usuario de la piedra en la arquitectura. Informstone. Bilbao, $399 \mathrm{pp}$.

Masana, E., Pallás, R., Perea, H., Ortuño,M., MartínezDíaz,J.J., García-Meléndez, E. and Santanach, P. 2005. Large Holocene morphogenic earthquakes along the Albox fault, Betic Cordillera, Spain. Journal of Geodynamics, 40, 119-133.

Martín, J.D. 2004. Using X-powder: a software package for powder Xray diffraction analysis, Version 2004.03. http://www.xpowder.com, 105 pp, Spain (L.GR1001/04.ISBN84-609-1497-6).

Martínez-Díaz, J.J. and Hernández Enrile, J.L. 2001. Uso de deformaciones de travertinos para caracterizar la actividad paleosísmica de una falla oblícua activa: la falla de Alhama de Murcia (Cordilleras Béticas, España). Acta Geológica Hispánica, 36, 297-313.

Martínez-Díaz, J.J., Rigo, A., Louis, L., Capote, R., Hernández-Enrile, J.L., Carreño E. and Tsige,M. 2002. Caracterización geológica y sismotectónica del terremoto de Mula (febrero de 1999, Mb: 4,8) mediante la utilización de datos geológicos, sismológicos y de interferometría de RADAR (INSAR). Boletín Geológico Minero, 113, 23-33.

Martínez-Martínez, J., Benavente, D. and García-del-Cura, M.A. 2011. Spatial attenuation: the most sensitive ultrasonic parameter for detecting petrographic features and decay processes in carbonate rocks. Engineering Geology, 19, 84-95.

Meijninger, B.M.L. 2007. Late-orogenic extension and strikeslip deformation in the Neogene of southeastern Spain. Geologica Ultraiectina. Mededelingen van de Faculteit Geowetenschappen, Universiteit Utrecht, No. 269, 176 pp.

Molina, E., Cultrone, J., Sebastián, E., Alonso, F. J., Carrizo, L., Gisbert, J. and Bug,O. 2011. The pore system of sedimentary rocks as a key factor in the durability of building materials. Engineering Geology, 118, 110-121.

Ordóñez, S., and García-del-Cura, M.A. 1983. Recent and 
tertiary fluvial carbonates in Central Spain. In. J. D. Collinson \& J. Lewin Eds Modern and Ancient Fluvial Systems. Spec. Publ. int. Sedimentologist, 6, Blackwell Scientific Publication. Oxford, 485-497.

Ordóñez, S., García-del-Cura, M. A., Fort, R. and Pina, J. A. 1996. El "Crema Marfil" (Pinoso Alicante) I. Características petrofísicas y parámetros de durabilidad. Geogaceta, 20 (3), 727- 730.

Ordóñez, S., González Martín, J.A., García-del-Cura, M.A. and Pedley, H.M. 2005. Temperate and semi-arid tufas in the Pleistocene to Recent fluvial barrage system in the Mediterranean area: the Ruidera Lakes Natural Park (Central Spain). Geomorphology, 69: 332-350.

Ozkul, M., Gokgoz, A.; Kele, S.; Oruc Baykara, M, ChuanChou Shen, Yu-Wei Chang, Kaya, A.; Mete Hancer, Aratman, C.; Akin; T and Zey Nep O. 2014. Sedimentological and geochemical characteristics of a fluvial travertine: A case from the eastern Mediterranean region. Sedimentology, 61, 291-318.

Pedley, M. 2009. Tufas and travertines of the Mediterranean region: a testing ground for freshwater carbonate concepts and developments. Sedimentology, 56, 221-46.

Pedley, M., González Martín, J.A., Ordóñez Delgado, S. and García-del-Cura, M.A. 2003. Sedimentology of Quaternary perched springline and paludal tufas: criteria for recognition, with examples from Guadalajara Province, Spain. Sedimentology, 50, 23-44.

Pentecost A. 2005. Travertine. Springer-Verlag; Berlín 445 pags.

Pentecost A, and Viles HA. 1994. A review and reassessment of travertine classification. Geogr Phys Quaternaire, 48, 305-14.

RocMaquina. 2012. La piedra Natural. Directorio Anual de la Piedra Natural. RocMaquina. Bilbao 352 pp.

Sanz de Galdeano, C., Galindo-Zaldívar, H., Alfaro, P. and Ruano, P. 2007. El relieve de la Cordillera Bética. Enseñanza de las Ciencias de la Tierra, 15-2, 185-195.

Silva, P.G., Mather, A.E., Goy, J.L., Zazo, C and Harvey, A.M. 1996. Controles en el desarrollo y evolución del drenaje en zonas tectónicamente activas: el caso del río Mula. (Región de Murcia, SE de España). Rev. Soc. Geol. España, 9 (3-4), 269-283.

Silva, P.G. and Rodríguez Pascua, M.A. (Eds) 2014. Catálogo de los efectos geológicos de los terremotos en España. IGME -AEQUA, Madrid, 358 pp.

Sánchez-Martos, F. 2001. Las aguas subterráneas en el Bajo Andarax (Almería). Monografías Ciencia y Tecnología 12. Universidad de Almería, 278 pp.

Soler Huertas, B. 2005. El travertino rojo de Mula (Murcia). Definición de un mármol local. Verdolay, 9, 141-164.

Török, A. 2008. Black crusts on travertine: factors controlling development and stability. Environmental Geology, 56, 583-594.

Török, A. and Vásárhelyi, B. 2010. The influence of fabric and water content on selected rocks mechanical parameters of travertine, examples from Hungary. Engineering Geology, 115, 237-245.

UNE-EN 1925. 1999. Métodos de ensayo para piedra natural. Determinación del coeficiente de absorción de agua por capilaridad. AENOR. Madrid, $13 \mathrm{pp}$.

UNE-EN 1926. 2007. Métodos de ensayo para piedra natural. Determinación de la resistencia a compresión uniaxial. AENOR. Madrid, $21 \mathrm{pp}$.

UNE-EN 1936. 2007. Métodos de ensayo para piedra natural. Determinación de la densidad real y aparente y de la porosidad abierta y total. AENOR. Madrid,

UNE-EN 14579. 2005. Métodos de ensayo para piedra natural. Determinación de la velocidad de propagación del sonido. AENOR. Madrid, $15 \mathrm{pp}$.

Urosevic, M., Sebastián Pardo, E. and Cardell, C. 2010. Rough and polished travertine building stone decay evaluated by a marine aerosol ageing test. Construction and Building Materials, 24, 1438-1448.

Urosevic, M. K., Sebastián Pardo, E., Ruiz-Agudo, E. and Cardell, C. 2011. Physical properties of carbonate rocks used as a modern and historic construction material in Eastern Andalusia, Spain. Materiales de Construcción, 61, 301, 93-114.

Vázquez-Urbez, M., Arenas, C. and Pardo, G. (2012). A sedimentary facies model for stepped, fluvial tufa systems in the Iberian Range (Spain): the Quaternary Piedra and Mesa valleys. Sedimentology, 59, 502-526.

Voermans, F., Simon, O.J., Martín García, L. and Gomez Prieto, J.A. 1972 Mapa Geológico de España 1:50.000, hoja $n^{\circ}$ 996, Huercal-Overa IGME, Ministerio de Industria y Energía, Madrid.

Recibido: diciembre 2015

Revisado: febrero 2016

Aceptado: abril 2016

Publicado: junio 2017 
